\title{
On locally self-similar fractional random fields indexed by a manifold
}

\author{
Jacques Istas*and Céline LACAUX ${ }^{\dagger}$
}

May 9, 2011

\begin{abstract}
Local self-similarity for Euclidean random fields has been introduced since a while. In this paper, we extend it for manifold indexed random fields. We then give examples and derive some properties of the tangent field. In the Gaussian $(\alpha=2)$ and $\alpha$-stable $(0<\alpha<2)$ cases, we obtain the expected relations between the fractional index $H$ and the stability index $\alpha$.
\end{abstract}

Keywords: Local self-similarity, random field, manifold.

Subject Classification MSC-2000: 60F05, 60G10, 60G18, 60G60.

\section{Introduction}

Self-similar random fields are widely used to model natural phenomena in Internet traffic, hydrology, geophysics or financial markets, e.g. [1, 13]. The most famous and classical self-similar model is the fractional Brownian motion, see $[7,8]$. However, self-similarity is a global property and is then too restrictive for some applications, see [12] and references therein. Therefore, $[2,9]$ has introduced a weakened property, called local asymptotically self-similarity (in short lass) at point $x_{0}$. This property has been defined for random fields indexed by the Euclidean space $\mathbb{R}^{n}$ and many examples have been developped to illustrate it, see [3] and references therein.

Observe that the self-similarity property is well-defined on Euclidean spaces but it is not on a metric space or even on a manifold equipped with a distance. Then, only weak versions of selfsimilarity, that still be global properties, have been defined, e.g. $[5,6]$. However, in this paper, we see that the local self-similarity, introduced for Euclidean fields in $[2,9]$, can also be extended in the manifold indexed field realm.

We then illustrate this property by some Gaussian and stable examples. Since moving average fractional fields are on common use for modelling purpose, see [11], we focus on spherical and hyperbolic moving average fractional fields. We also consider multifractional random fields. Then, we give some expected properties of tangent fields of lass random fields indexed by a manifold. Let us emphasize that in the manifold framework, tangent fields may not be defined on whole the tangent space and are self-similar but not at any scale. Moreover, if the manifold indexed field has weak stationary increments, so has the tangent field. Some expected consequences are derived. In particular, if the tangent field is Gaussian, then it is a restriction of an Euclidean fractional Brownian motion.

In Section 2, notation in the framework of manifold indexed lass random fields are given. Section 3 is devoted to the definition of the lass property. Examples are developed in Section 4. Then, some properties of the tangent field of a lass field are studied in Section 5.

\footnotetext{
${ }^{*}$ Laboratoire Jean Kuntzmann Université de Grenoble et CNRS F-38041 Grenoble Cedex 9. E-mail: Jacques.Istas@imag.fr

${ }^{\dagger}$ Institut Elie Cartan Nancy, Nancy-Université, CNRS, INRIA, Boulevard des Aiguillettes B.P. 239 F-54506 Vandœuvre lès Nancy.

E-mail: Celine.Lacaux@iecn.u-nancy.fr
} 


\section{Preliminaries and Notation}

Throughout this note, $(\mathcal{M}, g)$ is a $\mathcal{C}^{\infty}$ Riemannian manifold of dimension $n \in \mathbb{N} \backslash\{0\}$ and for any $M_{1}, M_{2} \in \mathcal{M}$, the distance $d\left(M_{1}, M_{2}\right)$ is the length of the shortest curve between $M_{1}$ and $M_{2}$.

Let $M_{0} \in \mathcal{M}$ and denote by $T_{M_{0}} \mathcal{M}$ the tangent space to $\mathcal{M}$ at $M_{0}$. Then, there exists a neighborhood $\mathcal{V}\left(M_{0}\right)$ of $M_{0}$ and $\delta \in(0,+\infty]$, see for example [4], such that

1. for all $M \in \mathcal{V}\left(M_{0}\right)$, there exists an unique minimal geodesic between $M$ and $M_{0}$,

2. the exponential map $\exp _{M_{0}}$ at point $M_{0}$ is a diffeomorphism between the open ball $\mathcal{B}(0, \delta) \subset$ $T_{M_{0}} \mathcal{M}$ and $\mathcal{V}\left(M_{0}\right)$.

In the following, $\Pi_{M_{0}}$ denotes the inverse of the exponential map $\exp _{M_{0}}$ at point $M_{0}$.

\section{$3 \quad$ Local asymptotic self-similarity property}

In the sense of $[2,9]$, a random field $(X(x))_{x \in \mathbb{R}^{n}}$ is locally asymptotically self-similar at point $x_{0}$ with index $H$ if

$$
\lim _{\varepsilon \rightarrow 0_{+}}\left(\frac{X\left(x_{0}+\varepsilon x\right)-X\left(x_{0}\right)}{\varepsilon^{H}}\right)_{x \in \mathbb{R}^{n}} \stackrel{(d)}{=}\left(Z_{x_{0}}(x)\right)_{x \in \mathbb{R}^{n}}
$$

where $\stackrel{(d)}{=}$ stands for equality of finite dimensional distributions and $Z_{x_{0}}$ is a non degenerate field. To extend this property to fields indexed by the manifold $\mathcal{M}$, we have to interpret $x_{0}+\varepsilon x$ as a point of $\mathbb{R}^{n}$ without the help of the addition. To this aim, let us observe that $x_{0}+\varepsilon x$ is the shift of $x_{0}$ by the vector $\varepsilon x \in T_{x_{0}} \mathbb{R}^{n} \approx \mathbb{R}^{n}$. Also, since the geodesics in $\mathbb{R}^{n}$ are the segments, $x_{0}+\varepsilon x=\exp _{x_{0}}(\varepsilon x)$. Then, we propose to replace in (1) the point $x_{0}$ by $M_{0}$ and its translate $x_{0}+\varepsilon x$ by

$$
M_{0}+\varepsilon v \stackrel{\text { def }}{=} \exp _{M_{0}}(\varepsilon v)
$$

Definition 3.1. A random field $X=(X(M))_{M \in \mathcal{M}}$ is locally asymptotically self-similar at point $M_{0}$ with index $H>0$ if

$$
\lim _{\varepsilon \rightarrow 0_{+}}\left(\frac{X\left(M_{0}+\varepsilon v\right)-X\left(M_{0}\right)}{\varepsilon^{H}}\right)_{v \in B(0, \delta)} \stackrel{(d)}{=}\left(Z_{M_{0}}(v)\right)_{v \in B(0, \delta)}
$$

with $M_{0}+\varepsilon v$ defined by (2), $\delta$ introduced in Section 2 and $Z_{M_{0}}$ a non degenerate field, which means that for almost all $\omega$, there exists $v$, such that $Z_{x_{0}}(v, \omega) \neq 0$. The random field $Z_{M_{0}}$ is called tangent field at point $M_{0}$ of $X$.

Remark 3.2. As one could expect, the definition 3.1 coincide with the definitions of [2, 9] in the framework of random fields indexed by $\mathbb{R}^{n}$ choosing $\delta=+\infty$.

Remark 3.3. Let us fix $M \in \mathcal{V}\left(M_{0}\right)$ and consider the unique $v \in \mathcal{B}(0, \delta)$ such that $M_{0}+v=$ $\exp _{M_{0}}(v)=M$. Then, $M_{0}+\varepsilon v$ describes the geodesic between $M_{0}$ and $M$ as $\varepsilon$ varies in $[0,1]$. In addition, $M_{0}+\varepsilon v$ tends to $M_{0}$ in the direction given by this geodesic as $\varepsilon$ tends to zero.

\section{Examples}

Let us now give examples of locally asymptotically self-similar random fields indexed by manifold $\mathcal{M}$, which may differ from the Euclidean space $\mathbb{R}^{n}$. Most of our examples are random fields indexed or by the $n$-dimensional unit sphere $\mathbb{S}_{n}=\left\{x_{1}, x_{2}, \ldots, x_{n+1} \in \mathbb{R}, \sum_{i=1}^{n+1} x_{i}^{2}=1\right\}$ or by the $n$-dimensional hyperbolic space $\mathbb{H}_{n}=\left\{x_{1}, x_{2}, \ldots, x_{n+1} \in \mathbb{R},-x_{1}^{2}+\sum_{i=2}^{n+1} x_{i}^{2}=1\right\}$. 


\subsection{Fractional Brownian motions indexed by a manifold}

Let $X_{H}=\left(X_{H}(M)\right)_{M \in \mathcal{M}}$ be a fractional Brownian motion indexed by the manifold $\mathcal{M}$ and with index $H$. This random field, introduced in the indexed manifold realm by [5], is a Gaussian centered random field such that

$$
\exists O \in \mathcal{M}, \forall(M, N) \in \mathcal{M}^{2}, \mathbb{E}\left(X_{H}(M) X_{H}(N)\right)=\frac{1}{2}\left(d^{2 H}(O, M)+d^{2 H}(O, N)-d^{2 H}(M, N)\right) .
$$

Moreover, $X_{H}$ exists for $H \in\left(0, \beta_{\mathcal{M}}\right]$, with $\beta_{\mathcal{M}}$ a constant depending on the manifold $\mathcal{M}$ (see $\left.[5,6]\right)$.

Let us now fix $M_{0} \in \mathcal{M}$ and $u, v \in \mathcal{B}(0, \delta) \subset T_{M_{0}} \mathcal{M}$. For every $\varepsilon \in(0,1]$, let

$$
R\left(M_{0}+\varepsilon v, M_{0}+\varepsilon w\right)=\varepsilon^{-2 H} \operatorname{Cov}\left(X_{H}\left(M_{0}+\varepsilon v\right)-X_{H}\left(M_{0}\right), X_{H}\left(M_{0}+\varepsilon w\right)-X_{H}\left(M_{0}\right)\right) .
$$

Then, using (3) and $d\left(M_{0}+u, M_{0}\right)=\|u\|$ for $u \in B(0, \delta)$, one easily obtains that

$$
R\left(M_{0}+\varepsilon v, M_{0}+\varepsilon w\right)=\frac{1}{2}\left(\|v\|^{2 H}+\|w\|^{2 H}-\frac{d^{2 H}\left(M_{0}+\varepsilon v, M_{0}+\varepsilon w\right)}{\varepsilon^{2 H}}\right)
$$

Let us now give the behaviour of $d\left(M_{0}+\varepsilon v, M_{0}+\varepsilon w\right)$ as $\varepsilon$ tends to $0_{+}$(see [10, Chapter 5]).

Lemma 4.1. For any $v, w \in \mathcal{B}(0, \delta)$, $\lim _{\varepsilon \rightarrow 0_{+}} \varepsilon^{-1} d\left(M_{0}+\varepsilon v, M_{0}+\varepsilon w\right)=\|v-w\|$.

Since $X_{H}$ is a centered Gaussian random field, one easily derives from the previous lemma that $X_{H}$ is locally asymptotically self-similar at point $M_{0}$ with index $H$ and with tangent field an Euclidean fractional Brownian motion $B_{H}$ with index $H$ restricted to $\mathcal{B}(0, \delta)$.

\subsection{Moving average fractional random fields}

Let $\alpha \in(0,2]$ and let $W_{\alpha, \mathcal{M}}$ be a symmetric real $\alpha$-stable random measure on the manifold $\mathcal{M}$ with control measure the uniform measure $\sigma_{\mathcal{M}}$ on $\mathcal{M}$ (see [11] for details on such random measures). Let us recall that the stochastic integral $\int_{\mathcal{M}} g d W_{\alpha, \mathcal{M}}$ is well-defined iff $g \in L^{\alpha}\left(\mathcal{M}, d \sigma_{\mathcal{M}}\right)$. Moreover, if $g \in L^{\alpha}\left(\mathcal{M}, d \sigma_{\mathcal{M}}\right)$, then

$$
\forall \lambda \in \mathbb{R}, \mathbb{E}\left(\mathrm{e}^{i \lambda \int_{\mathcal{M}} g d W_{\alpha, \mathcal{M}}}\right)=\exp \left(-|\lambda|^{\alpha} \int_{\mathcal{M}}|g|^{\alpha} d \sigma_{\mathcal{M}}\right) .
$$

Let $H \in \mathbb{R}$ such that $H \neq n / \alpha$ and $f: \mathbb{R}_{+} \rightarrow \mathbb{R}$ be a continuous function. As soon as

$$
X_{H, \alpha, \mathcal{M}}(M)=\int_{\mathcal{M}} d\left(M, M^{\prime}\right)^{H-n / \alpha} f\left(d\left(M, M^{\prime}\right)\right) d W_{\alpha, \mathcal{M}}\left(M^{\prime}\right), \quad M \in \mathcal{M},
$$

is well-defined, with convention $0^{\beta}=0$ for $\beta<0, X_{H, \alpha, \mathcal{M}}$ is called moving average fractional $\alpha$-stable random field. Note that $X_{H, 2, \mathcal{M}}$ is a Gaussian field.

To ensure that the singularity of the kernel at $M=M^{\prime}$ is given by $d\left(M, M^{\prime}\right)^{H-n / \alpha}$ we assume that $f(0) \neq 0$. Then for the sake of simplicity, we choose $f$ so that $f(0)=1$.

Let us now observe that $X_{H, \alpha, \mathcal{M}}(M)$ is well-defined iff

$$
I_{\mathcal{M}}(M):=\int_{\mathcal{M}} d\left(M, M^{\prime}\right)^{\alpha H-n}\left|f\left(d\left(M, M^{\prime}\right)\right)\right|^{\alpha} d \sigma_{\mathcal{M}}(M)<+\infty
$$

that is iff

$$
I_{\mathcal{M}}(M)=\int_{\mathbb{S}_{n-1}} \int_{0}^{\rho_{M}(\theta)} r^{\alpha H-1}|f(r)|^{\alpha} J_{M}(\theta, r) d r d \sigma_{\mathbb{S}_{n-1}}(\theta)<+\infty
$$

with $J_{M}$ the Jacobian of the exponential map $\exp _{M}$ at point $M$ and $\rho_{M}(\theta)$ the distance between $M$ and the cut locus in the direction $\theta$ (see [4]). The choice of $f$ is then closely linked to the behavior of $J_{M}$. Moreover, to prove the local asymptotic self-similarity, we use the dominated convergence theorem and then we may choose a quite simple function $f$. The next proposition state the behaviour of the increments around $M_{0}$. 
Proposition 4.2. Let $H>0$ such that $H \neq n / \alpha$. Assume that $\mathcal{M}=\mathbb{S}_{n}$ or $\mathbb{H}_{n}$. If $\mathcal{M}=\mathbb{H}_{n}$, we assume that $f(x)=\mathrm{e}^{-\gamma x}$. Observe that $\delta=\pi$ if $\mathcal{M}=\mathbb{S}_{n}$ and $\delta=+\infty$ if $\mathcal{M}=\mathbb{H}_{n}$.

1. (a) The random field $X_{H, \alpha, \mathbb{S}_{n}}$ is well-defined if and only if $H>0$.

(b) The random field $X_{H, \alpha, \mathbb{H}_{n}}$ is well-defined if and only if $(H>0$ and $\gamma>(n-1) / \alpha)$ or $(0<H<(n-1) / \alpha$ and $\gamma=(n-1) / \alpha)$.

2. Assume that $X_{H, \alpha, \mathcal{M}}$ is well-defined on $\mathcal{M}$ and that $f$ is $\mathcal{C}^{1}$. Define $g:[0,+\infty) \rightarrow \mathbb{R}$ by

$$
g(x)=x^{H-n / \alpha} f(x)
$$

with convention $0^{\beta}=0$ for $\beta<0$.

(a) If $H \in(0,1)$, then for every $M_{0} \in \mathcal{M}$,

$$
\lim _{\varepsilon \rightarrow 0_{+}}\left(\frac{X_{H, \alpha, \mathcal{M}}\left(M_{0}+\varepsilon v\right)-X_{H, \alpha, \mathcal{M}}\left(M_{0}\right)}{\varepsilon^{H}}\right)_{v \in B(0, \delta)} \stackrel{(d)}{=}\left(B_{H, \alpha}(v)\right)_{v \in B(0, \delta)},
$$

where $B_{H, \alpha}$ is an Euclidean moving average $\alpha$-stable random field with index $H$, that is

$$
B_{H, \alpha}(v)=\int_{\mathbb{R}^{n}}\left(\|v-x\|^{H-n / \alpha}-\|x\|^{H-n / \alpha}\right) W_{\alpha, \mathbb{R}^{n}}(d x) .
$$

(b) If $H>1$, then for every $M_{0} \in \mathcal{M}$,

$$
\lim _{\varepsilon \rightarrow 0_{+}}\left(\frac{X_{H, \alpha, \mathcal{M}}\left(M_{0}+\varepsilon v\right)-X_{H, \alpha, \mathcal{M}}\left(M_{0}\right)}{\varepsilon}\right)_{v \in B(0, \delta)} \stackrel{(d)}{=}\left(<v, S_{\alpha}>\right)_{v \in B(0, \delta)},
$$

where $S_{\alpha}$ is the the stable random variable defined by

$$
S_{\alpha}=\int_{\mathcal{M}} \frac{\Pi_{M_{0}}\left(M^{\prime}\right)}{\left\|\Pi_{M_{0}}\left(M^{\prime}\right)\right\|} g^{\prime}\left(d\left(M_{0}, M^{\prime}\right)\right) d W_{\alpha, \mathcal{M}}\left(M^{\prime}\right) .
$$

Proof. 1. Let us recall that $\rho(\theta):=\rho_{M}(\theta)=\delta$ and that

$$
J(r):=J_{M}(r, \theta)=\left\{\begin{aligned}
r^{1-n} \sin ^{n-1} r & \text { if } \mathcal{M}=\mathbb{S}_{n} \\
r^{1-n} \sinh ^{n-1} r & \text { if } \mathcal{M}=\mathbb{H}_{n}
\end{aligned}\right.
$$

Then, replacing in (6) $J_{M}$ and $\rho_{M}$ by their value, one easily obtains Assertion 1.

2. Let $M_{0} \in \mathcal{M}$ and consider the open ball $\mathcal{B}(0, \delta) \subset T_{M_{0}} \mathcal{M} \approx \mathbb{R}^{n}$. Then, let $\varepsilon \in(0,1], k \in \mathbb{N} \backslash\{0\}$, $\lambda=\left(\lambda_{1}, \ldots, \lambda_{k}\right) \in \mathbb{R}^{k}$ and $v=\left(v_{1}, \ldots, v_{k}\right) \in \mathcal{B}(0, \delta)^{k}$.

(a) Assume $H \in(0,1)$. Before we study the behavior of $X_{H, \alpha, \mathcal{M}}$ around $M_{0} \in \mathcal{M}$, let us observe that we can define the random field $Y_{H, \alpha, \mathcal{M}}$ on $\mathcal{M} \times \mathcal{M}$ by

$$
Y\left(M_{1}, M_{2}\right)=\int_{\mathcal{M}} d\left(M_{1}, M^{\prime}\right)^{H-n / \alpha} f\left(d\left(M_{2}, M^{\prime}\right)\right) d W_{\alpha, \mathcal{M}}\left(M^{\prime}\right), \quad M_{1}, M_{2} \in \mathcal{M}
$$

Then, $X_{H, \alpha, \mathcal{M}}\left(M_{0}+\varepsilon w\right)-X_{H, \alpha, \mathcal{M}}\left(M_{0}\right)=Z_{\varepsilon, 1}(w)+Z_{\varepsilon, 2}(w)$ with

$$
\left\{\begin{array}{l}
Z_{\varepsilon, 1}(w)=Y\left(M_{0}+\varepsilon w, M_{0}\right)-Y\left(M_{0}, M_{0}\right) \\
Z_{\varepsilon, 2}(w)=Y\left(M_{0}+\varepsilon w, M_{0}+\varepsilon w\right)-Y\left(M_{0}+\varepsilon w, M_{0}\right) .
\end{array}\right.
$$


We first study the behavior of $Z_{\varepsilon, 1}$ as $\varepsilon \rightarrow 0_{+}$. Using (4), the exponential map $\exp _{M_{0}}$ and the change of variables $\rho=r / \varepsilon$, one obtains that

$$
\mathbb{E}\left(\exp \left(i \varepsilon^{-H} \sum_{j=1}^{j} \lambda_{j} Z_{\varepsilon, 1}\left(v_{j}\right)\right)\right)=\exp \left(-\varepsilon^{-\alpha H} I_{1}(\varepsilon)\right)
$$

where $I_{1}(\varepsilon)=\int_{(0, \delta / \varepsilon) \times \mathbb{S}_{n-1}} f_{\varepsilon}(\rho, u) d \rho d \sigma_{\mathbb{S}_{n-1}}(u)$ with

$$
f_{\varepsilon}(\rho, u)=\varepsilon^{n}\left|\sum_{j=1}^{k} \lambda_{j}\left(d\left(M_{0}+\varepsilon v_{j}, M_{0}+\varepsilon \rho u\right)^{H-n / \alpha}-(\varepsilon \rho)^{H-n / \alpha}\right)\right|^{\alpha}|f(\varepsilon \rho)|^{\alpha} J(\varepsilon \rho) \rho^{n-1} .
$$

By definition of $J$ and continuity of $f$, Lemma 4.1 leads to

$$
\lim _{\varepsilon \rightarrow 0_{+}} \varepsilon^{-\alpha H} f_{\varepsilon}(\rho, u)=\left|\sum_{j=1}^{k} \lambda_{j}\left(\left\|v_{j}-\rho u\right\|^{H-n / \alpha}-\rho^{H-n / \alpha}\right)\right|^{\alpha} \rho^{n-1} .
$$

Moreover, using twice the triangle inequality and $d\left(M_{0}+\varepsilon w, M_{0}\right)=\varepsilon\|w\|$ (with $\|w\|<\delta$ ), one obtains that

$$
\varepsilon||\left|v_{j} \|-\rho\right| \leq d\left(M_{0}+\varepsilon v_{j}, M_{0}+\varepsilon \rho u\right) \leq \varepsilon\left(\left\|v_{j}\right\|+\rho\right) .
$$

Using this inequality, one establishes that $\sup _{\varepsilon \in(0,1]}\left|\varepsilon^{-\alpha H} f_{\varepsilon}\right| \leq G$ where $G$ is defined by $G(\rho, u)=c \rho^{n-1}\left[\sum_{j=1}^{k}\left|\lambda_{j}\right|\left(|||| v_{j}\left\|-\left.\rho\right|^{H-n / \alpha}-\rho^{H-n / \alpha}|++|||\left|v_{j} \|+\rho\right|^{H-n / \alpha}-\rho^{H-n / \alpha} \mid\right)\right]^{\alpha}\right.$.

with $c$ a finite positive constant. Since $H \in(0,1), G \in L^{1}\left((0,+\infty) \times \mathbb{S}_{n-1}, d \rho d \sigma_{\mathbb{S}_{n-1}}(u)\right)$ and the dominated convergence theorem implies that

$$
\lim _{\varepsilon \rightarrow 0_{+}} \mathbb{E}\left(i \exp \left(\sum_{j=1}^{k} \lambda_{j} Z_{\varepsilon, 1}\left(v_{j}\right)\right)\right)=\mathbb{E}\left(\exp \left(i \sum_{j=1}^{k} \lambda_{j} B_{H, \alpha}\left(v_{j}\right)\right)\right)
$$

with $B_{H, \alpha}$ defined by (8).

We now study the behavior of $Z_{\varepsilon, 2}$ as $\varepsilon \rightarrow 0_{+}$. For any $w \in B(0, \delta)$,

$$
\forall \lambda \in \mathbb{R}, \mathbb{E}\left(\exp \left(i \lambda \varepsilon^{-H} Z_{\varepsilon, 2}(w)\right)\right)=\exp \left(-|\lambda|^{\alpha} \varepsilon^{-\alpha H} I_{2}(\varepsilon)\right)
$$

with $I_{2}(\varepsilon)=\int_{\mathcal{M}} d\left(M_{0}+\varepsilon w, M^{\prime}\right)^{\alpha H-n}\left|f\left(d\left(M_{0}+\varepsilon w, M^{\prime}\right)\right)-f\left(d\left(M_{0}, M^{\prime}\right)\right)\right|^{\alpha} d \sigma_{\mathcal{M}}\left(M^{\prime}\right)$.

Using the mean value theorem and $H<1$, one checks that

$$
\lim _{\varepsilon \rightarrow 0_{+}} \varepsilon^{-H \alpha} I_{2}(\varepsilon)=0
$$

Therefore, for any $w \in B(0, \delta) \lim _{\varepsilon \rightarrow 0_{+}} \varepsilon^{-H} Z_{\varepsilon, 2}(u) \stackrel{(d)}{=} 0$ and Assertion 2(a) follows from Equation (10). 
(b) Assume that $H>1$. We only sketch the proof. Let $K=2 \max _{1 \leq j \leq k}\left\|v_{j}\right\|$. The main idea is to write $X_{H, \alpha, \mathcal{M}}(M)=X_{1, \varepsilon}(M)+X_{2, \varepsilon}(M)$ with

$$
X_{1, \varepsilon}(M)=\int_{\mathcal{M}} g\left(d\left(M, M^{\prime}\right)\right) \mathbf{1}_{d\left(M_{0}, M^{\prime}\right) \leq \varepsilon K} d \sigma_{\mathcal{M}}\left(M^{\prime}\right)
$$

and

$$
X_{2, \varepsilon}(M)=\int_{\mathcal{M}} g\left(d\left(M, M^{\prime}\right)\right) \mathbf{1}_{d\left(M_{0}, M^{\prime}\right)>\varepsilon K} d \sigma_{\mathcal{M}}\left(M^{\prime}\right)
$$

We recall that $g$ is defined by (7). Following the proof of Assertion 2(a), replacing $f$ by $f \mathbf{1}_{d\left(M_{0}, M^{\prime}\right) \leq \varepsilon K}$, one proves (even though $H>1$ ) that

$$
\lim _{\varepsilon \rightarrow 0_{+}}\left(\varepsilon^{-1}\left(X_{1, \varepsilon}\left(M_{0}+\varepsilon v\right)-X_{\varepsilon, 1}\left(M_{0}\right)\right)\right)_{v \in B(0, \delta)} \stackrel{(d)}{=} 0 .
$$

Let us now study the asymptotics of $X_{2, \varepsilon}$ as $\varepsilon$ tends to $0_{+}$. Let us write

$$
\sum_{j=1}^{k} \lambda_{j}\left(X_{\varepsilon, 2}\left(M_{0}+\varepsilon v_{j}\right)-X_{\varepsilon, 2}\left(M_{0}\right)\right)=\int_{\mathcal{M}} \tilde{g}_{\varepsilon}\left(M^{\prime}\right) d W_{\alpha, \mathcal{M}}\left(M^{\prime}\right)
$$

with

$$
\tilde{g}_{\varepsilon}\left(M^{\prime}\right):=\sum_{j=1}^{k} \lambda_{j}\left(g\left(d\left(M_{0}+\varepsilon v_{j}, M^{\prime}\right)\right)-g\left(d\left(M_{0}, M^{\prime}\right)\right)\right) \mathbf{1}_{d\left(M_{0}, M^{\prime}\right)>\varepsilon K} .
$$

For any $M^{\prime} \notin \operatorname{Cut}_{M_{0}} \cup\left\{M_{0}\right\}$, where $\operatorname{Cut}_{M_{0}}$ is the cut locus of $M_{0}$ in $\mathcal{M}$,

$$
\lim _{\varepsilon \rightarrow 0_{+}} \frac{d\left(M_{0}+\varepsilon w, M^{\prime}\right)-d\left(M_{0}, M^{\prime}\right)}{\varepsilon}=-\frac{\left\langle\Pi_{M_{0}}\left(M^{\prime}\right), w\right\rangle}{\left\|\Pi_{M_{0}}\left(M^{\prime}\right)\right\|} .
$$

Then, for any $M^{\prime} \notin \mathrm{Cut}_{M_{0}} \cup\left\{M_{0}\right\}$, a Taylor expansion leads to

$$
\tilde{g}\left(M^{\prime}\right)=\lim _{\varepsilon \rightarrow 0_{+}} \varepsilon^{-1} \tilde{g}_{\varepsilon}\left(M^{\prime}\right)=-\frac{g^{\prime}\left(d\left(M_{0}, M^{\prime}\right)\right)\left\langle\Pi_{M_{0}}\left(M^{\prime}\right), \sum_{j=1}^{k} \lambda_{j} v_{j}\right\rangle}{\left\|\Pi_{M_{0}}\left(M^{\prime}\right)\right\|} .
$$

Moreover applying the mean value Theorem to the function $g$ and noting that

$$
d\left(M_{0}+\varepsilon v_{j}, M^{\prime}\right) \geq d\left(M_{0}, M^{\prime}\right)-\varepsilon\left\|v_{j}\right\| \geq \frac{d\left(M_{0}, M^{\prime}\right)}{2}
$$

for $d\left(M_{0}, M^{\prime}\right) \geq \varepsilon K$, one finds $\tilde{G} \in L^{\alpha}\left(\mathcal{M}, d \sigma_{\mathcal{M}}\right)$ such that $\sup _{\varepsilon \in(0,1]}\left|\varepsilon^{-1} \tilde{g}_{\varepsilon}\right| \leq \tilde{G}$. Since $\sigma_{\mathcal{M}}\left(\mathrm{Cut}_{M_{0}} \cup\left\{M_{0}\right\}\right)=0$, the dominated convergence theorem then leads to

$$
\lim _{\varepsilon \rightarrow 0_{+}} \varepsilon^{-\alpha} \int_{\mathcal{M}}\left|\tilde{g}_{\varepsilon}\right|^{\alpha} d \sigma_{\mathcal{M}}=\int_{\mathcal{M}}|\tilde{g}|^{\alpha} d \sigma_{\mathcal{M}}
$$

Combining this with (4) and (11), one obtains that

$$
\lim _{\varepsilon \rightarrow 0_{+}}\left(X_{H, \alpha, \mathcal{M}}\left(M_{0}+\varepsilon v\right)-X_{H, \alpha, \mathcal{M}}\left(M_{0}\right)\right)_{v \in B(0, \delta)}=\left(-\left\langle v, S_{\alpha}\right\rangle\right)_{v \in B(0, \delta)}
$$

with $S_{\alpha}$ defined by $(9)$. Since $S_{\alpha} \stackrel{(d)}{=}-S_{\alpha}$, the proof of Proposition 4.2 is then complete. 


\subsection{Moving average multifractional random fields}

As done for Euclidean random fields, to obtain some fields whose index of lass property vary with $M_{0} \in \mathcal{M}$, we replace the index $H$ by a function $h(\cdot)$ in the integral representation (5). Hence, let us consider a function $h: \mathcal{M} \longrightarrow(0,+\infty)$ such that $h(M) \neq n / \alpha$ for every $M \in \mathcal{M}$. Then, as soon as

$$
X_{h, \alpha, \mathcal{M}}(M)=\int_{\mathcal{M}} d\left(M, M^{\prime}\right)^{h(M)-n / \alpha} f\left(d\left(M, M^{\prime}\right)\right) d W_{\alpha, \mathcal{M}}\left(M^{\prime}\right), M \in \mathcal{M}
$$

is well-defined, $X_{h, \alpha}$ is called moving average multifractional $\alpha$-stable random field with multifractional function $h$. Then, replacing $H$ by $h\left(M_{0}\right)$ and assuming $h$ sufficiently smooth, we can state the analoguous of proposition 4.2 for the random field $X_{h, \alpha, \mathcal{M}}$.

Proposition 4.3. Assume that $\mathcal{M}=\mathbb{S}_{n}$ or $\mathbb{H}_{n}$. If $\mathcal{M}=\mathbb{H}_{n}$, we assume that $f(x)=\mathrm{e}^{-\gamma x}$ with $\gamma>(n-1) / \alpha$. Then, $X_{h, \alpha, \mathcal{M}}$ is well-defined. Moreover, if $h$ and $f$ are $\mathcal{C}^{1}$ and if $h\left(M_{0}\right)<1$ for $M_{0} \in \mathcal{M}$, then,

$$
\lim _{\varepsilon \rightarrow 0_{+}}\left(\frac{X_{h, \alpha, \mathcal{M}}\left(M_{0}+\varepsilon v\right)-X\left(M_{0}\right)}{\varepsilon^{h\left(M_{0}\right)}}\right)_{v \in B(0, \delta)} \stackrel{(d)}{=}\left(B_{h\left(M_{0}\right), \alpha}(v)\right)_{v \in B(0, \delta)},
$$

where $B_{h\left(M_{0}\right), \alpha}$ is defined by (8).

Proof of proposition 4.3. By Proposition 4.2, the random field

$$
Y(M, H)=\int_{\mathcal{M}} d\left(M, M^{\prime}\right)^{H-n / \alpha} f\left(d\left(M, M^{\prime}\right)\right) d W_{\alpha, \mathcal{M}}\left(M^{\prime}\right), M \in \mathcal{M}, H>0
$$

is well-defined. For every $v \in \mathcal{B}(0, \delta)$ and every $\varepsilon \in(0,1)$, let us write that

$$
X_{h, \alpha, \mathcal{M}}\left(M_{0}+\varepsilon v\right)-X_{h, \alpha, \mathcal{M}}\left(M_{0}\right)=Y\left(M_{0}+\varepsilon v, h\left(M_{0}\right)\right)-Y\left(M_{0}, h\left(M_{0}\right)\right)+R_{M_{0}}\left(M_{0}+\varepsilon v\right)
$$

with $R_{M_{0}}(M)=Y_{\alpha, \mathcal{M}}(M, h(M))-Y_{\alpha, \mathcal{M}}\left(M, h\left(M_{0}\right)\right)$. Then, by (4),

$$
\mathbb{E}\left(\exp \left(i u R_{M_{0}}\left(M_{0}+\varepsilon v\right)\right)\right)=\exp \left(-|u|^{\alpha} I(\varepsilon)\right)
$$

with

$$
I(\varepsilon)=\frac{2 \pi^{n / 2}}{\Gamma(n / 2)} \int_{0}^{\delta}\left|r^{h\left(M_{0}+\varepsilon v\right)-n / \alpha}-r^{h\left(M_{0}\right)-n / \alpha}\right|^{\alpha}|f(r)|^{\alpha} d r .
$$

Let $\varepsilon_{0}>0$. Then, by continuity of $h$ there exists $a, b \in(0,+\infty)$ such that for $\varepsilon \in\left[0, \varepsilon_{0}\right), a \leq$ $h\left(M_{0}+\varepsilon v\right) \leq b$. Hence, applying the Taylor-Lagrange inequality, one obtains that for $\varepsilon \in\left(0, \varepsilon_{0}\right)$,

$$
I(\varepsilon) \leq \frac{2 \pi^{n / 2}}{\Gamma(n / 2)}\left|h\left(M_{0}+\varepsilon v\right)-h\left(M_{0}\right)\right|^{\alpha} \int_{0}^{\delta}|\ln r|\left(r^{a-n / \alpha}+r^{b-n / \alpha}\right)^{\alpha}|f(r)|^{\alpha} d r
$$

Since $h$ is a $\mathcal{C}^{1}$ function and $h\left(M_{0}\right)<1, \lim _{\varepsilon \rightarrow 0_{+}} \varepsilon^{-h\left(M_{0}\right)} R_{M_{0}}\left(M_{0}+\varepsilon v\right) \stackrel{(d)}{=} 0$. Then, one concludes the proof applying Proposition 4.2 to the moving average fractional $\alpha$-stable field $Y\left(\cdot, h\left(M_{0}\right)\right)$, whose index is $h\left(M_{0}\right)<1$.

\section{Classical properties of the tangent field}

We give in this section some general properties of fractional fields, especially properties of the tangent fields and range of permissible fractional index. These properties are indeed more or less expected.

The self-similarity, stated in next proposition, of the tangent field as small scales is a direct consequence of the definition of tangent fields. 
Proposition 5.1. If the random field $X=(X(M))_{M \in \mathcal{M}}$ is locally asymptotically self-similar random field at point $M_{0}$ with index $H$ and tangent field $Z_{M_{0}}$, then $Z_{M_{0}}(0)=0$ almost surely and

$$
\forall \lambda \in(0,1],\left(Z_{M_{0}}(\lambda v)\right)_{v \in B(0, \delta)} \stackrel{(d)}{=} \lambda^{H}\left(Z_{M_{0}}(v)\right)_{v \in B(0, \delta)} .
$$

Under a weak stationary property, defined below, the range of permissible indices $H$ depends on the existence of moments, with a break for moments higher that one. In the case of fields indexed by whole $\mathbb{R}^{n}$, this property has already been established in [11].

Definition 5.2. The increments of the field $X=(X(M))_{M \in \mathcal{M}}$ are weakly stationary if for all $(M, N) \in \mathcal{M}^{2}$, the distribution of $X(M)-X(N)$ only depends on the geodesic distance $d(M, N)$.

The fractional fields defined in Sections 4.1 and 4.2 are examples of fields with weakly stationary increments. Then, they illustrate the following proposition.

Proposition 5.3. Let $X$ be a locally asymptotically self-similar random field at point $M_{0}$ with index $H$ and tangent field $Z_{M_{0}}$. Assume that $X$ has weakly stationary increments.

1. Then, $Z_{M_{0}}$ has weakly stationary increments.

2. If for some $u \in \mathcal{B}(0, \delta) \backslash\{0\}$ and some $\gamma>0, \mathbb{P}\left(Z_{M_{0}}(u) \neq 0\right)=1$ and $\mathbb{E}\left|Z_{M_{0}}(u)\right|^{\gamma}<+\infty$, then $0<H<\max \left(\frac{1}{\gamma}, 1\right)$.

3. Assume $Z_{M_{0}}$ is a Gaussian random field. Then $H \in(0,1]$ and there exists a finite constant $C>0$ such

$$
\forall v, w \in \mathcal{B}(0, \delta), \mathbb{E}\left(Z_{M_{0}}(v) Z_{M_{0}}(w)\right)=\frac{C^{2}}{2}\left(\|v\|^{2 H}+\|w\|^{2 H}-\|v-w\|^{2 H}\right) .
$$

Moreover, if $H<1, Z_{M_{0}}$ is centered and is then an Euclidean fractional Brownian motion restricted to $B(0, \delta)$.

4. If $Z_{M_{0}}$ is an $\alpha$-stable random field, then $0<H \leq \max \left(1, \frac{1}{\alpha}\right)$.

These properties are already known for Euclidean random fields (see [11]) and then were expected in the manifold indexed realm. As regards Assertions 2-4, we provide a proof to emphasize where the manifold realm plays a role, and refer to [11] for some details (see proof of Proposition 7.1.10, Corollary 7.1.11 and Lemma 7.2.1).

Proof of Proposition 5.3.

1. Let $v, w \in \mathcal{B}(0, \delta)$. Then, by the lass property

$$
Z_{M_{0}}(v)-Z_{M_{0}}(w) \stackrel{(d)}{=} \lim _{\varepsilon \rightarrow 0_{+}} \frac{X\left(M_{0}+\varepsilon v\right)-X\left(M_{0}+\varepsilon w\right)}{\varepsilon^{H}} .
$$

Let us fix $u \in \mathcal{B}(0, \delta)$ such that $u \neq 0$. Then, by continuity of the distance and of the exponential map $\exp _{M_{0}}$, there exists $\varepsilon_{0}>0$ such that

$$
\forall \varepsilon \leq \varepsilon_{0}, d\left(M_{0}+\varepsilon v, M_{0}+\varepsilon w\right)=d\left(\exp _{M_{0}}(\varepsilon v), \exp _{M_{0}}(\varepsilon w)\right)<\|u\| .
$$

Therefore, for every $\varepsilon \leq \varepsilon_{0}$, the point

$$
M_{\varepsilon}=M_{0}+\frac{d\left(M_{0}+\varepsilon v, M_{0}+\varepsilon w\right)}{\|u\|} u=\exp _{M_{0}}\left(\frac{d\left(M_{0}+\varepsilon v, M_{0}+\varepsilon w\right)}{\|u\|} u\right)
$$


is well-defined and such that $d\left(M_{0}, M_{\varepsilon}\right)=d\left(M_{0}+\varepsilon v, M_{0}+\varepsilon w\right)$. Hence, by (15) and by the weakly stationarity of the increments of $X$,

$$
Z_{M_{0}}(v)-Z_{M_{0}}(w) \stackrel{(d)}{=} \lim _{\varepsilon \rightarrow 0_{+}} \frac{X\left(M_{\varepsilon}\right)-X\left(M_{0}\right)}{\varepsilon^{H}} .
$$

Observe that $M_{\varepsilon}=M_{0}+\varepsilon^{\prime} u$ where $\varepsilon^{\prime}=d\left(M_{0}+\varepsilon v, M_{0}+\varepsilon w\right) \rightarrow 0+$ as $\varepsilon \rightarrow 0_{+}$. Hence, applying again the lass property and Lemma 4.1, we obtain that

$$
Z_{M_{0}}(v)-Z_{M_{0}}(w) \stackrel{(d)}{=}\|u\|^{-H}\|v-w\|^{H} Z_{M_{0}}(u)
$$

Since this equality holds for any $v, w \in \mathcal{B}(0, \delta)$, the increments of $Z_{M_{0}}$ are weakly stationary.

2. Let us remark that $u / 2 \in \mathcal{B}(0, \delta)$ and then that $Z_{M_{0}}(u / 2)$ is well-defined. Then, since $Z_{M_{0}}(0)=$ 0 , by Equation (16) (applied $(v, w)=(u / 2,0)), Z_{M_{0}}(u / 2) \neq 0$ almost surely and by weak stationarity of the increments of $Z_{M_{0}}$,

$$
Z_{M_{0}}(u)-Z_{M_{0}}\left(\frac{u}{2}\right) \stackrel{(d)}{=} Z_{M_{0}}\left(\frac{u}{2}\right)
$$

Therefore, $\mathbb{P}\left(Z_{M_{0}}\left(\frac{u}{2}\right) \neq 0, Z_{M_{0}}(u) \neq Z_{M_{0}}\left(\frac{u}{2}\right)\right)=\mathbb{P}\left(Z_{M_{0}}(u) \neq Z_{M_{0}}\left(\frac{u}{2}\right)\right)=1>0$. Then, one can now follow the proof of Proposition 7.1.10 in [11] to establish Assertion 2 (replacing $X(2)-X(1)$ by $\left.Z_{M_{0}}(u)-Z_{M_{0}}\left(\frac{u}{2}\right)\right)$.

3. Assume that $Z_{M_{0}}$ is a centered Gaussian random field. We can not directly proceed as in the proof of Lemma 7.2.1 of [11] since $Z_{M_{0}}$ is not defined on whole $\mathbb{R}^{n}$. However, the idea stills be the same.

Let us first remark that since $Z_{M_{0}}$ is a non degenerate Gaussian random field, it fulfills the assumptions of Assertion 2 with $\gamma=2$, which implies that $H \leq 1$. Moreover, by Equation (16) (where we recall that $u \neq 0$ is a fix chosen point of $B(0, \delta)$ ), for every $v, w \in \mathcal{B}(0, \delta)$,

$$
\mathbb{E}\left(Z_{M_{0}}(v)-Z_{M_{0}}(w)\right)^{2}=C^{2}\|v-w\|^{2 H}
$$

with $C=\|u\|^{-H} \sqrt{\mathbb{E}\left(Z_{M_{0}}(u)\right)^{2}} \in(0,+\infty)$. Since $Z_{M_{0}}(0)=0$ almost surely, this is equivalent to (14). If $H<1$, using the self-similarity property (13) with $\lambda=1 / 2$ and the weak stationarity property, one checks, as in the proof of Lemma 7.2.1 in [11], that

$$
\mathbb{E}\left(Z_{M_{0}}(u / 2)\right)=\left(2^{-H}-1\right) \mathbb{E}\left(Z_{M_{0}}(u)\right) .
$$

This implies that $\mathbb{E}\left(Z_{M_{0}}(u)\right)=0$ and then by (16) that $Z_{M_{0}}$ is centered.

4. Since $Z_{M_{0}}$ is an $\alpha$-stable random field, it fullfils the assumptions of Assertion 2 for any $\gamma<\alpha$ and then $H \leq \max (1 / \alpha, 1)$.

\section{References}

[1] P. Abry, P. Goncalves, and J. Lévy Véhel. Lois d'échelle, fractales et ondelettes, volume 1. Hermes, 2002.

[2] A. Benassi, S. Jaffard, and D. Roux. Gaussian processes and Pseudodifferential Elliptic operators. Revista Mathematica Iberoamericana, 13(1):19-89, 1997. 
[3] S. Cohen and J. Istas. Fractional fields and applications. 2011. Monography, submitted.

[4] S. Gallot, D. Hulin, and J. Lafontaine. Riemannian geometry. Universitext. Springer-Verlag, Berlin, second edition, 1990.

[5] J. Istas. Spherical and Hyperbolic Fractional Brownian Motion. Elec. Comm. Prob., 10:254-262, 2005.

[6] J. Istas. On fractional stable fields indexed by metric spaces. Elec. Comm. Prob., 11:242-251, 2006.

[7] A. N. Kolmogorov. Wienersche Spiralen und einige andere interessante Kurven in Hilbertsche Raum. C. R. (Dokl.) Acad. Sci. URSS, 26:115-118, 1940.

[8] B. B. Mandelbrot and J. Van Ness. Fractional Brownian motion, fractional noises and applications. Siam Review, 10:422-437, 1968.

[9] R. F. Peltier and J. Lévy Véhel. Multifractional Brownian motion: definition and preliminary results. available on http://hal.inria.fr/inria-00074045/fr/, 1996.

[10] P. Petersen. Riemannian geometry, volume 171 of Graduate Texts in Mathematics. SpringerVerlag, New York, 1998.

[11] G. Samorodnitsky and M. S. Taqqu. Stable non-Gaussian random processes. Stochastic Modeling. Chapman \& Hall, New York, 1994. Stochastic models with infinite variance.

[12] S. Stoev, M. S. Taqqu, C. Park, G. Michailidis, and J. S. Marron. LASS: a tool for the local analysis of self-similarity. Comput. Statist. Data Anal., 50(9):2447-2471, 2006.

[13] W. Willinger, V. Paxson, and M. S. Taqqu. Self-similarity and heavy tails: Structural modeling of network traffic. In A practical guide to heavy tails (Santa Barbara, CA, 1995), pages 27-53. Birkhäuser Boston, Boston, MA, 1998. 